\title{
El método de enseñanza bilingüe de inmersión parcial y el método de intensificación para la enseñanza aprendizaje de inglés en las capacidades generales y específicas en estudiantes de sexto grado
}

\author{
The method of bilingual partial immersion teaching and the method of \\ intensification for the teaching of English in general and specific skills in sixth \\ grade students
}

Roxana Cardich San Juan ${ }^{1}$

\begin{abstract}
RESUMEN
El estudio busca determinar los efectos que ejercen en el desarrollo de las capacidades cognitivas de los estudiantes en dos tipos de programas de enseñanza para la lengua extranjera, como lo representan el programa bilingüe de inmersión parcial frente a un programa bilingüe de intensificación en la enseñanza de un segundo idioma. El estudio se realiza con 130 estudiantes que cursan el sexto grado de primaria. Los resultados muestran diferencias globales en las capacidades generales y específicas de los alumnos evaluados con un mejor desempeño del grupo que estudia con una metodología de intensificación. El estudio distingue diferencias en las áreas de atención y concentración, adaptación, exactitud lectora, ortografía fonética, cálculo y resolución de problemas matemáticos destacando con mejores puntuaciones comparativas los alumnos que estudian con una metodología de intensificación. Las áreas que no muestran diferencias son el razonamiento comprensión lectora, velocidad lectora y la ortografía visual y reglada. La investigación conduce a valorizar la importancia de la enseñanza de una segunda lengua desde la educación primaria, mediante el empleo de metodologías comunicativas que respeten la consolidación de la lengua materna en el aprendizaje y fomente la vinculación con un segundo idioma de manera utilitaria, práctica y vivencial.
\end{abstract}

\section{Palabras clave}

Capacidades generales, capacidades específicas, bilingüismo, método bilingüe de inmersión parcial, método de intensificación en la enseñanza-aprendizaje de una segunda lengua.

\begin{abstract}
The study seeks to determine the effects that two training programs types for foreign language as represented by the partial immersion bilingual program to a program of bilingual education intensification of a second language have on the development of student cognitive abilities,. The study has been carried out with 130 students in the sixth grade of elementary school. The results show global differences in the general and specific abilities of the evaluated student with a better performance of the group that studies with a methodology of intensification. The study distinguishes differences in the areas of attention and concentration, adaptation, reading accuracy, spelling phonics, arithmetic and mathematical problem solving, standing out comparatively higher scoring students who study following a method of intensification. The areas that show no differences are the reason reading comprehension, reading speed and visual and regulated spelling. The research leads to value the importance of teaching a second language from primary education through the use of communicative methodologies that respect the consolidation of the mother language in learning and encourage the linkage with a second language in a utilitarian, practical and experiential way.
\end{abstract}

Magister en Psicología Educativa y Doctorando de la Universidad Nacional Mayor de San Marcos. Cuenta con experiencia en el área educativa y psicológica, en especial en el diseño e implementación de programas psicopedagógicos y en el asesoramiento de trabajos de investigación. 


\section{Keywords}

General abilities, specific abilities, bilingualism, bilingual partial immersion method, second language intensifying teaching-learning method.

\section{INTRODUCCIÓN}

Las características de una sociedad cada vez más competitiva y globalizada demandan a las escuelas el desarrollo en sus estudiantes de diferentes habilidades a temprana edad. Es por ello, que no es extraño que en la actualidad en gran parte de las instituciones educativas, se desarrollen programas cada vez más sofisticados y rigurosos dirigidos al manejo de las tecnologías de la información y las comunicaciones, se implementen cursos destinados a promover tanto habilidades personales como sociales y se consoliden además sistemas de enseñanza de idiomas como el inglés, el francés, el alemán, el italiano $e$ incluso el chino bajo diferentes modalidades y estrategias de aprendizaje.

Carecería de relevancia, analizar la importancia que se debe brindar a la preparación personal y académica de los niños para hacer frente a las exigencias de la sociedad actual, como tampoco sería pertinente discutir la trascendencia que posee la enseñanza de un segundo idioma en la escuela. En este sentido, es indiscutible que el aprendizaje y el desarrollo de una lengua extranjera amplía notoriamente el conocimiento y posibilidades de educación, facilitando la sensibilidad cultural al mismo tiempo que garantiza una mayor comprensión de otras formas de pensamiento, permitiendo así alcanzar una visión globalizada $e$ integradora del mundo.

Sin embargo, resulta interesante profundizar en el análisis de las metodologías para la enseñanza de un segundo idioma, las cuales se espera busquen promover y al mismo tiempo garantizar el óptimo desarrollo de habilidades lingüísticas en dos lenguas: la materna y la extranjera. Esta reflexión surge principalmente, al profundizar en los procesos de aprendizaje propios de los programas de educación inicial y primaria de un sistema bilingüe, en especial, cuando se trabajan planes curriculares dirigidos a desarrollar a una temprana edad, algunas capacidades cognitivas que permitan analizar, comprender, resolver problemas y producir textos a temprana edad. Cabe resaltar que específicamente en esta investigación se profundiza en la comparación de los efectos en las capacidades cognitivas y adaptativas de dos tipos de metodologías de aprendizaje de un segundo idioma. La primera denominada método bilingüe de inmersión parcial que involucra un sistema de enseñanza en donde se utiliza el segundo idioma como método de instrucción en la mayoría de materias académicas. El objetivo central que persigue este método es la alfabetización completa y el desarrollo de estrategias comunicativas tanto en la lengua materna como en la nueva lengua. Así, en el programa de inmersión parcial, por ejemplo, el aprendizaje de la lectoescritura se hace de manera simultánea a partir del primer grado de primaria (Genesee, Lambert y Holobow, 1986, p. 32). La segunda metodología es la denominada por De Mejía y Fonseca (2009, p. 26) Método de intensificación en la Enseñanza-Aprendizaje. Esta última hace referencia a los programas que dedican en promedio entre diez a quince horas semanales al aprendizaje de una segunda lengua extranjera como materia sin que esta se use como medio de aprendizaje en ningún área curricular. Es decir, se destaca el desarrollo de competencias comunicativas principalmente en la nueva lengua a través de su aprendizaje como materia.

Estas inquietudes en el estudio del bilingüismo se dirigen a esclarecer los efectos en el aprendizaje y en el desarrollo de capacidades de los niños cuando se encuentran inmersos en una segunda lengua de manera constante a través de las distintas materias académicas. Esta preocupación se acrecienta cuando se toma consciencia de que en los años de enseñanza primaria se busca prioritariamente desarrollar diversas capacidades expresadas en el desarrollo de estrategias que permitan al niño desarrollar su pensamiento reflexivo y crítico expresado en la solución de problemas lógicos, verbales y adaptativos en un medio en constante transformación. Como línea 
de análisis de esta problemática, De Mejía y Fonseca (2009, p. 29) alerta de que una de las tendencias actuales en los colegios bilingües es brindar mayor importancia a la lengua extranjera antes que al español, asumiendo que este segundo idioma no necesita trabajo intencional mayor por hallarse naturalmente en el medio escolar de los niños. Ante esta situación, vale la pena preguntarse qué puede ocurrirle al nivel del desarrollo del español en los países latinoamericanos, si las habilidades lingüísticas en este idioma no se manejan en la mayoría de las áreas del currículo escolar. Esta interrogante cobra mayor sentido al destacar lo propuesto por Cummins (1983, p. 47), quien plantea que el nivel de competencia en un segundo idioma dependerá en parte del tipo de competencia que se haya logrado en la lengua materna.

Es pertinente destacar que en diversas investigaciones realizadas sobre el tema de bilingüismo, se enfatiza que el aprendizaje de un segundo idioma favorece el desarrollo lingüístico y cognitivo (Cansigno, 2006, p. 2). Sin embargo, los críticos a esta posición plantean que estos estudios se han desarrollado en contextos de inmigración o con variables poco controladas. Al respecto, Vila (1983, p. 6) señala que no se cuenta con un riguroso soporte científico que permita obtener una teoría global de la educación bilingüe, a pesar del interés que ha suscitado el tema tanto en lingüistas, pedagogos, psicólogos y sociólogos. Los especialistas en esta línea de investigación insisten en enriquecer la reflexión y la discusión del bilingüismo especialmente en educación.

Algunos aspectos relevantes que no se pueden obviar al abordar el bilingüismo son los referidos a los procesos que intervienen en el aprendizaje de una segunda lengua. En tal sentido, Navarro (2010, p. 121), enfatiza que para entender la educación bilingüe, se debe tener en cuenta que el aprendizaje de la lengua materna se realiza de manera inconsciente y espontánea, mientras que en el caso de la segunda lengua el aprendizaje se realiza a través de la instrucción entendida como una planificación del aprendizaje y enseñanza, con una metodología concreta. Arias Lozano (2000, p. 122) destaca que tanto en la educación bilingüe como en la regular se debe enfatizar en la expresión de los contenidos bajo un sistema comunicativo activo, antes de acceder a la lengua escrita. Este, sin duda, constituye un eslabón y un gran aporte del método comunicativo para el aprendizaje de los idiomas en general.

Es preciso destacar que, en el marco del proceso de enseñanza-aprendizaje de una segunda lengua en el nuevo milenio, se presentan como una alternativa educativa los programas de inmersión para el aprendizaje de una segunda lengua, los mismos que tuvieron sus orígenes en Canadá. Entre las ventajas competitivas que ofrecen estos programas se destacan la interacción significativa en el idioma entre los estudiantes y mayores oportunidades de una corrección compartida. En la actualidad, diversos centros educativos en el mundo optan por esta opción desde edades tempranas como una alternativa para que sus estudiantes puedan adquirir un alto dominio de diferentes idiomas. Otras posturas más conservadoras concuerdan que los programas de inmersión no constituyen el único camino para aprender otros idiomas y desarrollan diferentes propuestas metodológicas sin descuidar el valor del aprendizaje de una segunda o una tercera lengua y considerando sobre todo su edad de inicio.

Si bien los programas de inmersión han demostrado un sustantivo éxito en el desarrollo de las competencias lingüísticas de los niños para el desarrollo de un segundo idioma en contextos como Norteamérica y Europa, se debe destacar la necesidad de profundizar sus efectos y repercusiones en realidades latinoamericanas, dado que se observan pocos estudios rigurosos en esta parte del continente.

En este contexto, la investigación que se presenta está orientada a analizar y comparar las fortalezas y debilidades en el desarrollo de las capacidades generales como el razonamiento, los procesos de atención y memoria, los niveles de adaptación así como las repercusiones en el desarrollo de las capacidades específicas entre ellas la lectura, la escritura y el aprendizaje matemático de dos programas de enseñanza de un segundo idioma anteriormente 
descritos: el Método de Inmersión Parcial y en el segundo caso el Método Intensificación. Así, el Método Bilingüe de Inmersión Parcial hace referencia a un sistema de enseñanza, en el cual se utiliza el mismo idioma extranjero como método de instrucción en diferentes áreas académicas. Mientras que el Método de Intensificación focaliza la enseñanza de un segundo idioma en el fortalecimiento de habilidades comunicativas en esa lengua, sin incluir su aprendizaje a través de contenidos, cursos o materias académicas específicas.

Cabe destacar, que este trabajo se orienta a resaltar la importancia del aprendizaje bilingüe, pero además a esclarecer comparativamente los principales efectos de dos tipos de metodologías de enseñanza sobre el desarrollo de capacidades comprensivas de interacción y comunicación en la lengua materna, es decir, en el idioma español. El planteamiento de este estudio guarda una relación con los postulados que señalan que el hecho de ser bilingüe implica adoptar, comprender y respetar el sistema simbólico de otra cultura y al mismo tiempo interactuar con ella, considerando además que la educación bilingüe debe aprovechar las capacidades cognitivas del niño desarrollándose de manera natural y fundamentalmente como instrumento de comunicación (Cansigno, 2006, p. 5).

\section{MATERIAL Y MÉTODO}

La investigación es de tipo no experimental, descriptiva comparativa, utilizando un diseño transversal.

\section{Sujetos:}

La muestra estuvo constituida por 130 estudiantes de sexto grado de primaria, pertenecientes a dos colegios particulares mixtos del distrito de Lima. Se trabajó con dos grupos de niños de acuerdo al colegio de procedencia. El primer grupo caracterizado por desarrollar una metodología de inmersión parcial en el idioma inglés estuvo conformado por 64 estudiantes. El segundo grupo, dirigido a la enseñanza del idioma inglés a través del método de la intensificación estuvo conformado por 66 estudiantes. Dado el tamaño de la población, no se consideró conveniente utilizar alguna técnica para obtener el tamaño de la muestra, decidiendo por el trabajo con el $100 \%$ de los estudiantes del grado mencionado.

\section{Instrumento}

Para la recolección de datos se han considerado dos instrumentos: Una Ficha de Cotejo y la Batería Psicopedagógica EVALÚA-5 (Versión 2.0).

\section{A. Ficha de Cotejo:}

Este instrumento fue elaborado a partir de la investigación realizada por De Mejía (2009 p. 24 -26) en relación al documento titulado "Orientaciones para Políticas Bilingües y Multilingües enLenguas Extranjerasen Colombia". $\mathrm{Su}$ principal objetivo es evaluar las características de la metodología de enseñanza del idioma inglés como segundo idioma, para identificar el tipo de método de enseñanza. Cabe indicar que esta herramienta proporciona información sobre la orientación socio cultural de la metodología empleada, el número de horas de enseñanza en la segunda lengua, el tipo de textos utilizados, como adicionalmente la nacionalidad de la plana docente bilingüe de la institución.

Como criterios de evaluación se consideran: La acreditación, el número de horas de enseñanza del idioma inglés, los materiales de enseñanza, la orientación de la enseñanza en relación a la promoción de la cultura peruana como la extranjera. la orientación de los estudiantes en relación a realizar estudios en el extranjero, las visitas o pasantías al exterior y la nacionalidad de la plana docente.

\section{B. Batería Psicopedagógica Evalúa 5 (Versión 2.0)}

Ficha técnica:

Título del Instrumento:

Batería

Psicopedagógica Evalúa 5

(Versión 2.0) 
Año:

País:

Institución:

Forma de Aplicación:

Forma de Calificación : Corrección manual.

Objetivo: La Batería Psicopedagógica Evalúa 5 es un instrumento de evaluación psicopedagógica destinado a evaluar las capacidades cognoscitivas de los estudiantes que se encuentran finalizando el quinto grado o iniciando el sexto grado de primaria. Esta prueba está orientada a obtener datos relevantes para la toma de decisiones respecto a los procesos educativos a seguir en los centros educativos (García, González y García 2005, p. 11-23).

Contenido de la Prueba: En el contexto de una estrategia global de evaluación educativa, la Batería Psicopedagógica Evalua 5 propone aportar información relevante respecto a las siguientes variables:

Bases cognoscitivas del aprendizaje: Una parte importante de la batería se centra en el examen de procesos psicológicos comúnmente considerados como determinantes en la adquisición de nuevos conocimientos y habilidades como son la atención, la memoria, la capacidad de reflexionar (pensamiento inductivo analógico).

Adquisiciones instrumentales básicas: La prueba examina las adquisiciones y aspectos como la eficacia lectora, la comprensión de la lengua y expresión escrita, el cálculo numérico y la solución de problemas.

Aspectos afectivos y conductuales: brinda información sobre ciertas variables conductuales, socio-afectivas y actitudinales.

\section{Estructura de la Prueba}

\section{Capacidades Generales}

Bases del razonamiento: Sevalora el rendimiento en las tareas que exigen procesos de observación analítica, comparación, pensamiento analógico y organización perceptiva.
Reflexibilidad: Se explora la capacidad para seguir instrucciones, como comprobar su veracidad y falsedad mediante instrucciones. Pensamiento analógico: Se contempla la capacidad de inferir relaciones verbales a partir de las relaciones del mismo tipo identificadas previamente por observación y comparación. Organización Perceptiva: Se valorala capacidad para completar figuras, seleccionado la parte que falta entre varias alternativas.

Memoria-atención: Se examina la capacidad para mantener la atención focalizada en tareas que exigen observación analítica. Además, se explora la memoria a corto plazo en tareas de reconocimiento.

Niveles de adaptación: La batería incluye un índice global de adaptación, en base a la combinación de cuatro aspectos: Actitud Motivación (atracción o rechazo frente a las tareas escolares); Autocontrol y Autonomía (autonomía personal, la autopercepción en relación a posibles dificultades y la autorregulación); Conductas Pro-Sociales (disposición a la interacción cooperativa y de ayuda a sus pares).

\section{Capacidades Específicas}

Lectura: Donde se aprecia el nivel comprensivo como la eficacia de la lectura y escritura.

Comprensión lectora: La puntuación global de la prueba es un índice obtenido a través de diversas sub pruebas tales como: comprensión del vocabulario, realización de sencillas inferencias no explícitas en el texto $e$ identificación de las ideas principales.

Exactitud lectora: Se examina la eficacia del estudiante para interpretar mensajes orales y escritos a través del uso de la vía fonológica.

Velocidad lectora. Se ofrece un índice global obtenido a partir del análisis de la rapidez lectora alcanzada en un texto y de las respuestas en relación a la comprensión del mismo.

Escritura: La puntuación global de esta prueba es un resumen de los resultados obtenidos de los siguientes aspectos: ortografía fonética, grafía y expresión escrita, ortografía visual y reglada.

\section{Aprendizajes Matemáticos}

Se diferencian dos pruebas:

Cálculoy numeración. Explora el conocimiento de los números inferiores a un millón, aspectos 
relacionados con las secuencias numéricas y las diferencias de valor entre números así como la adquisición de los automatismos de la suma, resta, multiplicación, división y el concepto de fracción.

Resolución de problemas. Se obtiene a partir de la ejecución de diversos problemas aritméticos, formulados de modo que la dificultad básica sea la comprensión de un problema y la adecuada selección del procedimiento

\section{Normalización, Confiabilidad y Validez}

El proceso de elaboración de la Batería Evalúa 5 , a decir de sus autores ha tenido básicamente las siguientes fases $y$ procesos:

1ra. Fase: Elaboración y experimentación de la prueba "piloto" para delimitar cuestiones como: instrucciones, tiempos y configuración.

2da Fase. Elaboración y experimentación de la prueba pre experimental. A partir de la cual se consideró: la reducción de pruebas seleccionando índices de dificultades, orden de los ítems y configuración de la prueba experimental.

3ra. Fase. Elaboración y experimentación de la prueba experimental.

Para comprobar la fiabilidad de las pruebas, la dificultad, discriminación y varianza de los ítems, se empleó el programa informático Metrix.

Los datos más relevantes en la experimentación de la Batería Psicopedagógica Evalúa 5, son los siguientes:

1. Buena correlación con los contrastes externos establecidos, especialmente con el rendimiento escolar obtenido por los estudiantes durante el mismo curso escolar en que se realizó la aplicación experimental. Se obtuvo un coeficiente de Pearson 0,706 con un nivel de significación del 0.001 .

2. La correlación de las diferentes pruebas de la batería con el mismo rendimiento escolar refieren:

Bases del Razonamiento

0,709
Memoria y Atención

0,682

Comprensión Lectora

0,719

Cálculo

0,798

En lo que se refiere a la prueba de adaptación escolar, la correlación no es tan buena, dado que el coeficiente obtenido ha sido mucho más bajo, quizás debido al carácter del procedimiento empleado.

3. Un buen nivel de homogeneidad entre las pruebas y los bloques de pruebas que se compone la Batería Evalúa 5 que puede observar en los coeficientes de correlación de Pearson obtenidos al ubicar en relación los resultados alcanzados por cada bloque con las pruebas de que se componen.

a. Bases del razonamiento/pensamiento analógico

0,712

b. Bases del razonamiento/organización perceptiva $\quad 0,693$

c. Bases del razonamiento/reflexibilidad 0,719

Además de los estudios correlacionados, los autores han llevado un análisis de los ítems de cada una de las pruebas a fin de confeccionar la prueba definitiva mediante el ajuste de la fiabilidad y validez interna de cada una de las pruebas que compone la batería actual. Este análisis ha permitido: eliminar algunas tareas poco válidas, discriminar del número de ítems de algunas pruebas y reorganizar los ítems en todas las pruebas de la batería.

Considerando el material original, para fines de esta investigación se realizaron algunas modificaciones en cuanto a algunos ítems considerando la idiosincrasia de la cultura peruana.

\section{RESULTADOS}

Los resultados de este estudio, buscan comparar los efectos de dos metodologías de enseñanza de un segundo idioma: inmersión parcial y el método de intensificación sobre las capacidades generales, especificas cognitivas 
y adaptativas de dos grupos de estudiantes del sexto grado de educación básica. Para ello, se inició el proceso de calificación de resultados, elaborando una base de datos en el programa Excel, la misma que facilitó el obtener puntuaciones por preguntas, áreas y una tabulación final en la que se consideraron la conversión de puntajes directos. Para realizar la comprobación de hipótesis se utilizó el programa estadístico SSPS- 15. Se consideraron en primer término, datos descriptivos por cada grupo, teniendo referencia cada área e ítem evaluado por sujeto participante, para posteriormente aplicar las pruebas de normalización Smirnov Kolmogorov y la prueba estadística no paramétrica U de Mann Whitnney.

Las capacidades generales y específicas evaluadas son la atención y memoria, el razonamiento, la adaptación, la comprensión, la exactitud y velocidad lectora, la ortografía visual y reglada, el cálculo y la capacidad para resolver problemas matemáticos

Los resultados que se presentan en los siguientes párrafos se iniciarán a partir de la determinación de la normalidad estadística de los datos obtenidos, para desarrollar luego analíticamente las contrastaciones entre los grupos estudiados.

Con el propósito de verificar si los datos obtenidos corresponden a la normal estadística se ha calculado para cada factor medido, el coeficiente Z-K de SmirnovKolmogorov, encontrando resultados variantes entre normales y no normales, razón por la que se decide trabajar con estadísticos no paramétricos. Al respecto, se opta por utilizar es estadístico U Mann de Whitnney. Los resultados se presentan a continuación:

Tabla 1.

Cálculo de la Prueba U de Mann - Whitney en relación a las capacidades evaluadas al comparar la metodología de inmersión parcial y el método de intensificación

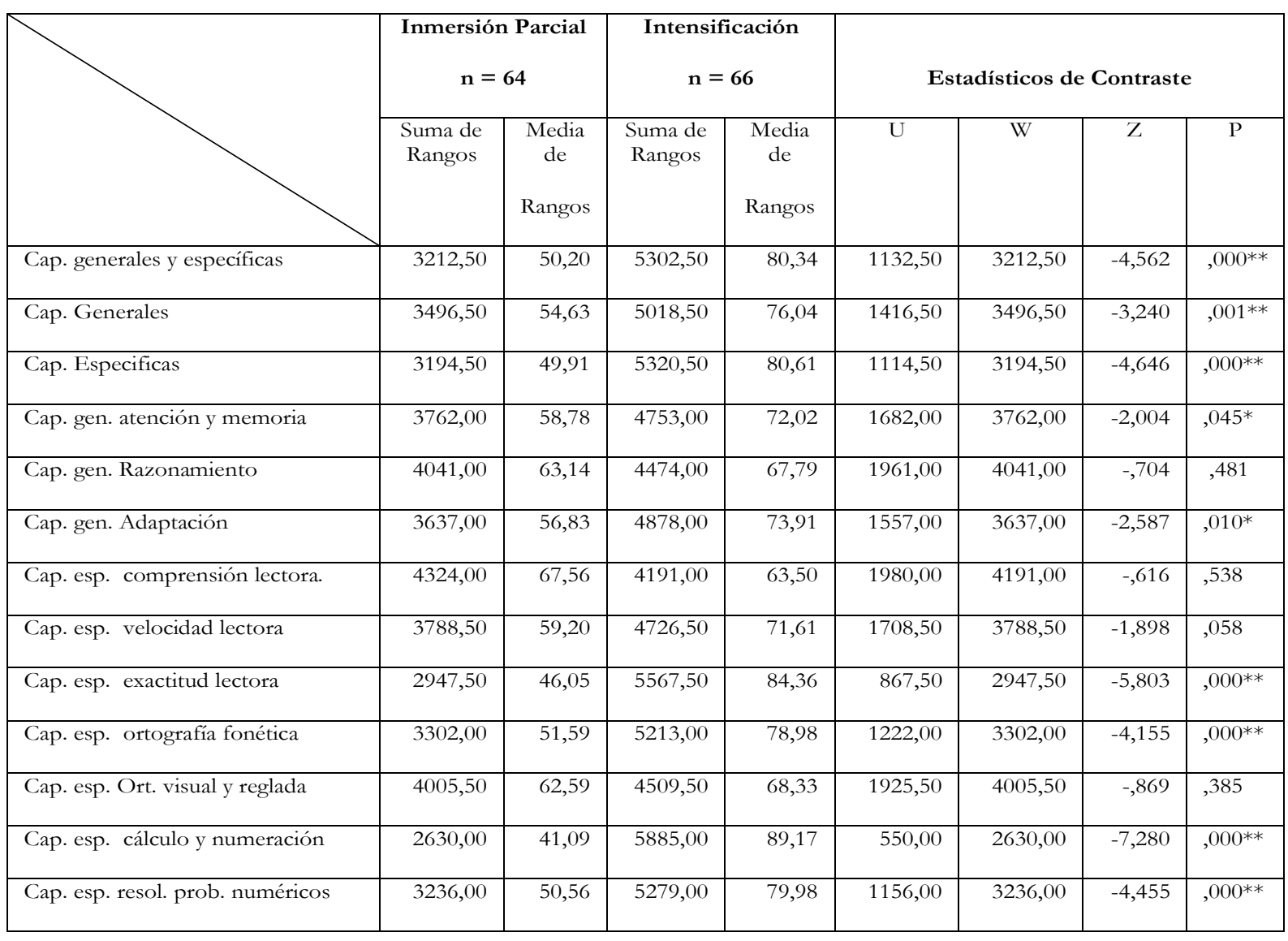

(*) $\quad \mathrm{P}<0.05$

$(* *) \mathrm{P}<0.01$ 
Al analizar los resultados, se observa comparativamente que en términos globales las puntuaciones referidas a las capacidades generales como específicas obtenidas por los grupos evaluados muestran diferencias significativas $(\mathrm{P}=0,000 ; \mathrm{P}<0,05)$, evidenciándose puntuaciones superiores en el grupo de estudiantes cuyo aprendizaje se realiza bajo el método de intensificación. Este hallazgo, permite aceptar la Hipótesis General del estudio, la cual plantea diferencias entre las capacidades generales y las capacidades específicas cognitivas y adaptativas de los estudiantes de sexto grado de primaria cuando se aplica el método de enseñanza bilingüe de inmersión parcial en comparación con los resultados obtenidos por los estudiantes que estudian el idioma inglés con el método de intensificación.

Cuando se realiza un análisis diferenciado entre las capacidades generales de ambos grupos, se obtiene una puntuación $\mathrm{P}$ equivalente a 0,001 $(P<0,05)$, resultado que evidencia diferencias entre ambos grupos cuando se considera de manera global las puntuaciones en la evaluación de las capacidades de atención y memoria, razonamiento y adaptación. Asimismo, se puede hacer referencia que al comparar los resultados obtenidos de manera integral a partir de la evaluación de las capacidades específicas de ambos grupos también se distinguen diferencias al obtenerse una $\mathrm{P}=0,000(\mathrm{P}<0,05)$. Esto significa, que al considerarse las puntuaciones globales de las capacidades específicas referidas a la comprensión, velocidad y exactitud lectora, ortografía fonética, visual y reglada, como el cálculo y la resolución de problemas matemáticos se aprecian diferencias entre los resultados obtenidos por ambos grupos, destacando con mejores puntuaciones comparativas los alumnos que estudian con una metodología de intensificación. Si bien estos resultados son interesantes, se hace necesario delimitar la diferenciación de las puntuaciones por áreas, para lo cual se realiza el análisis a partir de las tres capacidades generales: Atención - Memoria, Razonamiento y Adaptación.

Para el área de atención y memoria, la cual explora la capacidad para mantener la concentración en tareas que exigen observación analítica, como la capacidad de memoria a corto plazo en tareas de reconocimiento, se ha obtenido la puntuación P equivalente a 0,045 , evidenciándose la existencia de diferencias entre ambos grupos $(\mathrm{P}<0,05)$. Ante este criterio evaluado, el grupo que ofrece un mejor desempeño es el grupo que estudia con la metodología de enseñanza de intensificación.

Para la capacidad de razonamiento, de acuerdo con el instrumento seleccionado evalúa 5 , el rendimiento de los estudiantes en tareas que exigen procesos de observación analítica, pensamiento analógico y organización perceptiva, los resultados señalan una puntuación $\mathrm{P}$ equivalente a $0,481 \quad(\mathrm{P}>0,05)$ condición que indica la no existencia de diferencias entre ambos grupos para esta área.

Al explorar los resultados obtenidos para el área de Adaptación, la cual contempla la valoración de la motivación del alumno hacia el trabajo escolar, como también su capacidad de autocontrol y autonomía, como las conductas prosociales entendidas como la disposición del alumno a la interacción con sus pares y el autoconcepto, los resultados señalan la existencia de diferencias significativas entre ambos grupos $(\mathrm{P}=0,010 ; \mathrm{P}<0,05)$.

Se puede destacar en base a los análisis estadísticos realizados que las áreas en las que los alumnos muestran diferencias significativas son las que corresponden al área de autoconcepto y autoestima. Sin embargo, no se distinguen diferencias en los aspectos de motivación, autocontrol y conductas prosociales.

Los resultados presentados, para la variable Comprensión lectora señalan una puntuación $\mathrm{P}$ igual a $0,538(\mathrm{P}>0,05)$ condición que refleja la no existencia de diferencias significativas entre grupos. En otras palabras, de acuerdo a las evaluaciones realizadas, cuando los estudiantes de diferente metodología de enseñanza deben enfrentarse a tareas que involucren comprensión de vocabulario, así como la realización de sencillas inferencias no implícitas en un texto, o la identificación de las ideas principales, no se aprecian evidencias 
que señalen diferencias entre sus desempeños. La velocidad lectora permite conjugar el desempeño de los estudiantes en términos de rapidez para la lectura, como la capacidad comprensiva alcanzada en la prueba. Al analizar estadísticamente los resultados de los grupos evaluados se obtiene una puntuación $\mathrm{P}$ equivalente a 0,058 ( $\mathrm{P}>0,05)$. Se concluye que al evaluar la velocidad lectora en relación al desempeño de los estudiantes que utilizan diferente metodología para la enseñanza del idioma inglés, no se distinguen diferencias significativas.

Sin embargo, los resultados alcanzados en exactitud lectora demuestran la presencia de diferencias entre los grupos evaluados. Se llega a este hallazgo al obtener una $\mathrm{P}$ igual a $0,000(\mathrm{P}<0,05)$. Así, cuando se examina la eficacia lectora de los alumnos para interpretar mensajes orales y escritos, haciendo uso de la vía fonológica el grupo que obtiene un mejor desempeño es el conformado por los estudiantes del programa de intensificación.

Para la evaluación de la ortografía fonética, los resultados se han establecido prioritariamente a través de la copia y el dictado de diferentes tareas, pretendiéndose que a partir de ellas se evalúe la calidad ortográfica de lo escrito identificando posibles errores como omisiones o inversiones. Las puntuaciones en esta área le brindan ventaja al grupo cuya metodología es la propia del programa de intensificación. Se obtiene una $\mathrm{P}=0,000(\mathrm{P}<0,05)$.

Cuando se evalúa la calidad ortográfica en lo que se refiere a distinguir errores ortográficos de carácter visual y la transgresión de reglas ortográficas se obtiene una puntuación $\mathrm{P}=$ $0,385$ ( $P>0,05)$, condición que destaca la no diferenciación entre los grupos.

Además, los resultados observados en cálculo y numeración, expresan diferencias entre los grupos, ofreciendo un mejor desempeño del grupo que cuenta con una metodología de intensificación para el aprendizaje del idioma inglés. Esto permite plantear, que ante tareas en las que se busca valorar el conocimiento de los aspectos relacionados con las secuencias numéricas, las diferencias de valor entre números, como la adquisición de los automatismos de las operaciones básicas, existen diferencias significativas $(P=0,000 ; P<0,05)$ entre ambos grupos.

Cuando se examina la probabilidad de diferencias en la capacidad para resolver problemas matemáticos que involucran la comprensión de problemas y la adecuada selección del procedimiento de resolución se concluye que esta afirmación es positiva $(\mathrm{P}=0,000 ; \mathrm{P}<0,05)$, observándose un mejor desenvolvimiento del grupo que estudia bajo una metodología de enseñanza de intensificación.

\section{DISCUSIÓN}

El problema presentado en esta investigación representa un tema que ha generado en el marco de la educación y la psicolingüística diversas posiciones a lo largo de las últimas décadas. Ciertamente ante este estudio, se encuentran posiciones favorables que destacan sobre todo la valoración en el desarrollo de las capacidades cognitivas y la flexibilidad mental en los estudiantes que se inician tempranamente en una educación bilingüe. Otras posiciones frente al mismo problema, expresan preocupación en el desarrollo comprensivo del lenguaje, sobre todo cuando se inicia un programa bilingüe en condiciones que la lengua materna no se encuentra completamente consolidada, destacándose además como un argumento sensible de análisis, el hecho que la enseñanza temprana de un segundo idioma no garantiza la efectividad en su aprendizaje. En esta orientación, se enfatiza la necesidad de no perder de vista que las ventajas para iniciar el aprendizaje de un segundo idioma deben ser superiores a las posibles interferencias lingüísticas.

Se debe enfatizar que este estudio, no pretende negar la importancia de la enseñanza de un segundo idioma en la escuela, pero sí busca contribuir a establecer un mayor conocimiento de los efectos de dos de las múltiples metodologías que se emplean actualmente en algunos de los colegios bilingües, así como esclarecer sus posibles efectos en el desarrollo 
de las capacidades de los estudiantes sobre todo a temprana edad.

En tal sentido, esta investigación comparte el criterio de Cummins (1983, p. 47), quien sostiene que el aprendizaje de un segundo idioma, contribuirá a promover la formación actitudinal y cognitiva de los estudiantes respetando la idea que el nivel de competencia en la segunda lengua de un niño, dependerá del tipo de competencia que haya logrado en su lengua materna.

En relación a los resultados expuestos de manera global, se destaca la existencia de diferencias en las capacidades generales y en las capacidades específicas de los estudiantes evaluados. Específicamente, en lo que corresponde a capacidades generales se puede concluir que la metodología de enseñanza de intensificación para la enseñanza de inglés ofrece mejores resultados en comparación e la metodología de inmersión parcial en las áreas de atención y concentración como en el área de adaptación.

Estos primeros hallazgos, en relación a las capacidades generales de los grupos, ameritan nuevas investigaciones las cuales se orienten a profundizar en los posibles efectos de las metodologías de enseñanza y de la capacidad de atención y concentración por una parte. Asimismo, considerando que los resultados han sido manejados de manera global para la capacidad de adaptación, se hace necesario establecer nuevos estudios tratando de delimitar los aspectos referidos a la autoestima $e$ incluso a la valoración de la autoeficacia de los alumnos que se encuentran inmersos en los programas tempranos para la adquisición de lenguas extranjeras.

Al valorar el aspecto de lectura evaluado por la prueba seleccionada, se destaca que en las áreas de comprensión y velocidad lectora no se distinguen diferencias significativas, lo que indicaría que estos dos tipos de metodología de enseñanza no afectarían el desempeño de los estudiantes en éstas áreas. No obstante, al valorizar dos áreas relacionadas con el manejo fonético del lenguaje se pueden apreciar datos interesantes. Tanto para la capacidad de exactitud lectora, (que contempla la eficacia lectora para interpretar mensajes a través de la vía fonológica mediante la utilización de palabras parecidas fonológicamente), como para la capacidad de ortografía fonética, se distinguen la diferenciación significativa del desempeño de ambos grupos. Este dato, podría interpretarse como una posible interferencia del segundo idioma en el aprendizaje de las tareas fonéticas en la lengua materna, considerándose que el grupo que utiliza una metodología en la que sus cursos básicos de aprendizaje se realizan en el idioma inglés su rendimiento se encuentra disminuido en comparación con el segundo tipo de enseñanza.

Otra capacidad sensible que evidenciaría los efectos de la enseñanza de un idioma extranjero en el aprendizaje, es la que corresponde al área numérica o también llamada en el marco educativo para el nivel primario en el área lógico matemática. En tal sentido, los alumnos vinculados a un sistema de enseñanza de inmersión parcial evidencian un rendimiento disminuido tanto en el aspecto de cálculo como en la resolución de problemas numéricos. Estos resultados, ciertamente generan la necesidad de profundizar en algunos aspectos específicos propios de esta capacidad, en tal sentido se sugiere analizar a través de otros estudios, los efectos del tipo de metodología empleada, como incluso los materiales de enseñanza cuando se emplea un segundo idioma en la enseñanza de la matemática como materia.

Los resultados obtenidos en las capacidades de cálculo y resolución de problemas numéricos también conducen a cuestionar la efectividad de la enseñanza del curso de matemática completamente en una lengua extranjera. En relación con este postulado, si se contempla la hipótesis del umbral, la cual expone que deben existir niveles de competencias lingüísticas que el niño debe poseer en ambas lenguas para poder alcanzar con éxito los logros de una educación bilingüe, entonces bien valdría considerar el desarrollo de habilidades prioritariamente en la lengua materna antes de una exposición completa en un segundo idioma en esta área de aprendizaje. Los hallazgos presentados, guardarían relación con lo postulado con Malavé (2003, p. 4), quien plantea que el rendimiento para la matemática 
se encuentra supeditado al desarrollo en el estudiante de las destrezas de proficiencia cognoscitiva y académica principalmente en el idioma en que va a resolver el problema matemático. En tal sentido, al desarrollarse el aprendizaje del curso en un segundo idioma sin considerar variables tales como los esquemas sociales, lingüísticos, culturales y cognoscitivos se establecerían limitaciones en el desempeño cognitivo de esta materia. Una aproximación y salida al problema estaría vinculada a analizar dichas variables y considerarlas de manera integral desde el currículo educativo.

Dados estos resultados, la investigación conduce ciertamente a valorizar la importancia de la enseñanza de una segunda lengua desde la educación primaria, sin embargo esta debe dirigirse y direccionarse hacia el empleo de metodologías novedosas y pertinentes como la constituye el programa de enseñanza dual dentro de un enfoque comunicativo, la misma que respete la consolidación de la lengua materna en el aprendizaje y fomente la vinculación con un segundo idioma de manera utilitaria, funcional, práctica y vivencial.

\section{CONCLUSIONES}

- El trabajo de investigación presentado, destaca la importancia de analizar las metodologías más adecuadas para desarrollar una educación bilingüe en la formación básica regular, considerando su relevancia en función de las demandas y las implicaciones de la sociedad actual.

- Es reconocible que en el análisis de la problemática del estudio del bilingüismo, existen planteamientos favorables relacionados con la valoración del desarrollo de las capacidades cognitivas, como posturas antagónicas, que cuestionan sus repercusiones, sobre todo en el desarrollo comprensivo del lenguaje.

- La investigación teórica del tema evidencia, que en los países latinoamericanos, se presenta poca uniformidad en los planteamientos que se emplean en la educación básica, sobre todo para la enseñanza de un idioma extranjero.

- Las hipótesis del estudio se dirigen a comparar las capacidades generales y específicas cognitivas y adaptativas en español de estudiantes de sexto grado que desarrollan sus estudios en base a dos tipos de metodologías para la enseñanza de una segunda lengua: la metodología de intensificación en la que los alumnos estudian la segunda lengua diariamente pero sin intervención en cursos básicos y la metodología de inmersión parcial, en donde los cursos básicos son desarrollados en el segundo idioma.

- El estudio utiliza como instrumento de evaluación la prueba EVALUA - 5, la misma que considera la valoración de las capacidades generales tales como atención y memoria, razonamiento, y adaptación. Además, el test empleado distingue como capacidades específicas la comprensión, velocidad, exactitud lectora, la ortografía fonética, la ortografía visual y reglada como el cálculo y la resolución de problemas matemáticos.

- Los resultados globales de la investigación, señalan diferencias entre las capacidades generales y específicas cognitivas y adaptativas en español entre los dos grupos de estudiantes. Las puntuaciones globales obtenidas revelan que los estudiantes a los que se enseña con una metodología de intensificación obtienen mejores puntuaciones cuando se les compara con el grupo de estudiantes del mismo grado que estudia con una metodología de inmersión parcial.

- Al examinar las áreas cognitivas evaluadas como capacidades generales, se detectan diferencias significativas en la correspondiente al área de atención y memoria. No 
ocurre lo mismo con el razonamiento y los procesos de observación analítica, pensamiento analógico y organización perceptiva. En relación al aspecto adaptativo se observan diferencias en relación a los valores de autoconcepto y autoestima. Sin embargo, no se aprecian diferencias en los aspectos de motivación, autocontrol y conductas prosociales. Cabe resaltar, que los resultados que consignan diferencias, señalan ventajas en las puntuaciones de los estudiantes con metodología de intensificación.

- Al realizar un análisis de las capacidades específicas se aprecian diferencias en las áreas de exactitud lectora y ortografía fonética. Estos resultados, condicionados por la influencia fonológica del idioma, evidenciarían que cuando se examina la eficacia lectora para interpretar mensajes orales y escritos, como cuando se evalúa la calidad ortográfica de lo escrito, existen diferencias a favor de la metodología de intensificación.

- Las metodologías de enseñanza, no expresan diferencias en las áreas que involucran la comprensión de textos, incluso cuando es evaluada contemplando el tiempo (velocidad lectora), como cuando se examina el manejo ortográfico utilizando el canal visual.

- Se distinguen además diferencias significativas al contemplar las capacidades de cálculo y numeración como la capacidad resolución de problemas matemáticos, evidenciándose que los alumnos que estudian con una metodología de inmersión, la cual incluye la enseñanza de la matemática en un segundo idioma, muestran puntuaciones disminuidas, en comparación con los estudiantes que estudian esta asignatura en español.

\section{AGRADECIMIENTOS}

Un agradecimiento muy especial, al Doctor Luis Vicuña, quien asesoró este trabajo de investigación. 


\section{REFERENCIAS}

Arias Lozano, Leonora Patricia (2000) Habilidades Psicolingüísticas: Un estudio comparativo entre niños hispano hablantes mestizos y zapotecos. Recuperado el 19 de mayo de 2009. Desde: www.uam.mx/cdi2jornadas/2125.html.

De Mejía, Anne Marie y Tejada, Harvey (2001) La construcción de una propuesta curricular bilingüe para colegios monolingües en Cali. Universidad del Valle.

De Mejía, Anne Marie y colab. (2006) Estudio investigativo sobre el estado actual de la educación bilingüe (inglés- español) en Colombia. Ministerio de Educación Nacional. Centro de Investigación y Formación en Educación. Universidad de los Andes.

De Mejía, Anne Marie (2009) Orientaciones para políticas bilingües y multilingues en lenguas extranjeras en Colombia. Centro de Investigación y Formación en Educación. Universidad de los Andes. Bogotá.

Cummins, Jim. (1983) Interdependencia lingüística y desarrollo educativo en niños bilingües. Revista Infancia y Aprendizaje. Universidad de Alberta. Recuperado el 05 de julio de 2009. Desde: www.dialnet.unirioja.es.

Casigno Gutiérrez, Yvonne (2006) El bilingüismo en los contextos lingüístico y cognitivo. Relingüística Nro. 4. Lengua y Cultura. Recuperado el 13 de septiembre Desde: http://relinguistica. azc.uam.mx.

García, Jesús, Gonzalez, Daniel y García Beatriz (2005) Coordinador General: Miguel Martínez Batería. Psicopedagógica EVALUA 5. (Versión 2.0). Instituto de Orientación Psicológica EOS. Madrid.

Genesee, Fred, Lambert, William y Holobow, Naomi (1986) Universidad de Mc Gill. Traducción: Seminario de Bilingüismo de la Universidad Complutense. Revista Infancia y Aprendizaje. 33 27-36. Extraído el 13 de agosto de 2017. Desde: https://dialnet.unirioja.es.

Malavé, Liliam (2003) Fundamentos cognoscitivos: La enseñanza del inglés como segundo idioma. Universidad de Búfalo. Recuperado el 30 de junio de 2009. Desde: www.redewnlaces.ucv.cl.

Navarro Romero, Betsabé (2010) Adquisición de la primera y segunda lengua en aprendientes en edad infantil y adulta. Revista Semestral de Iniciación a la Investigación en Filología Vol 2. Universidad de Almería. Recuperado el 22 de junio 2011. Desde:www.ual.es/revistas.

Vila Ignasi (1983) Reflexiones en torno al bilingüismo y la enseñanza bilingüe. Universidad de Barcelona. Revista Infancia y Aprendizaje. Nro. 21. Recuperado el 16 de septiembre de 2009. Desde: www.dialnet.unirioja.es.

\section{BIBLIOGRAFÍA}

Akerberg, Marianne (2005) Adquisición de segundas lenguas Estudios y perspectivas. Líneas de Investigación en el Departamento de Lingüística Aplicada. Universidad Autónoma de México.

Albert, M y Obler, L (1978) The bilingual brain. Academic Press. Cambridge.

Alarcón Neve, Luisa (1998) El fenómeno del bilingüismo y sus implicaciones en el desarrollo 
cognitivo del individuo. Colección Pedagogía Universitaria N. ${ }^{\circ}$ 29. Universidad Veracruzana, México. Recuperado el 12 de junio de 2009. Desde: http://www.uv.mx/cpue/coleccion.

Areizaga, E (2003) La interculturalidad en el aula de español como lengua. Textos de Didáctica de la Lengua y de la Literatura Nro. 34. Recuperado el 08 de julio de 2009. Desde: www.dialnet. unirioja.es.

Arnau y colab. (1992) La educación bilingüe. Barcelona. Ice Horsori.

Artigal, José María (1990) Uso y adquisición de una lengua extranjera en el marco escolar entre los tres y los seis años. Revista Comunicación, Lenguaje y Educación. España.

Ascencio, Milton (2005) Adquisición de una segunda lengua en el salón de clases; ¿Subconsciente o consciente? Recuperado el 23 de marzo de 2011. Desde: http://www.udb.edu.sv/dialogos

Banfi, Cristina y Rettaroli, Silvia (2007) El bilingüismo y la educación bilingüe en Argentina. Revista Internacional Magisterio. Educación y Pedagogía. Bogotá.

Benitez Menéndez, Orquidea. (2007) Las tareas comunicativas en el aprendizaje de lenguas extranjeras: una alternativa para el desarrollo de habilidades comunicativas. Nro. 42/5. Revista Ibero Americana de Educación. Extraído el 22 de marzo de 2011. Desde: http://www.rieoei.org/ experiencias150.htm

Ben Xeev, S. (1981) Influencia del bilingüismo en la estrategia cognitiva y el desarrollo cognitivo. Revista Estudios de Psicología. Editores Infancia y Aprendizaje. España.

Berko Cleason, Jean y Bernstein, Nan (1999) Psicolinguística. Mc Graw Hill. Madrid.

Blanco Abarca, Amalio. (1981) Bilingüismo y cognición. Universidad Autónoma de Madrid. Estudios de Psicología Nro. 8.

Borrego y colab. (2000) Cuestiones de actualidad en lengua española. Instituto Caro y Cuervo. Ediciones Universidad Salamanca.

Bustinza Sovero, Telvy y Crosby Herrera, Jeannette (1995) Nivel de desarrollo de la expresión oral de los niños de cinco años de edad pertenecientes a un contexto bilingüe y monolingüe: estudio realizado en los centros educativos iniciales estatales del distrito de Ate-Vitarte. Tesis de Licenciatura. UNIFE Facultad de Educación. Programa de Educación Inicial. Lima.

Cenoz, Jasone (1997) La adquisición de la tercera lengua: bilingüismo y plurilingüismo en el País Vasco. AILE 10.

Cenoz, Jasone (2003) El Aprendizaje del inglés desde educación infantil: Efectos cognitivos, lingüísticos y afectivos. Universidad del País Vasco.

Córdova G, Paola. (2006) ¿Cambio o muerte de las lenguas? Reformas sobre la diversidad lingüística, social y cultural del Perú. Fondo Editorial de la Universidad de Ciencias Aplicadas. Lima.

Cortada de Kohan, Nuria (2005) Posibilidad de integración de las teorías cognitivas y la psicometría moderna. Interdisciplinaria. Vol.22, no.1. Recuperado el 20 de julio de 2009. Desde: http:www. scielo.org.ar. 
Corrrales Wade, Kathleen. (2009) Construyendo un segundo idioma. El constructivismo y la enseñanza del L2. Zona Próxima. Nro. 10. Universidad del Norte. www.redalyc.uaemex.mex.

Crawford. (2008) Early education and development. Recuperado el 20 de mayo de 2009 desde: www.parom.convio.nit/site.

Cummins, J y Swain, M (1986) Bilingualism in education. London: Longman.

Cummins, Jim. (2002). Lenguaje poder y pedagogía. Ediciones Morata. Madrid.

De Zubiría, Juan. (2007) ¿Tiene razón el Ministerio de Educación Nacional para adoptar el bilingüismo? Recuperado el 26 de julio de 2009. Desde: www.institutomerani.edu.co.

Dorsch (1985) Diccionario de Psicología. Barcelona, Herder.

Edel Navarrro, R. (2000) La educación y el desarrollo de habilidades cognitivas. Revista de la Universidad Cristóbal Colón Nro. 17-18. Extraído el 30 de septiembre de 2009 desde: www. eumed.net/rev.

Escobar, Alberto (1972) El multilingüismo en el Perú. Instituto de Estudios Peruanos. Lima.

Escobar, Alberto y colab. (1975) Perú ¿País bilingüe? Instituto de Estudios Peruanos. Lima.

Escobar, Ana María (1988) Hacia una tipología del bilingüismo en el Perú. Documento de trabajo Nro. 28. Serie Lingüística Nro. 2. Instituto de Estudios Peruanos. Lima.

Escutia López, Marciano. (2004) Perspectiva psicolingüística del bilingüismo. Universidad Complutense de Madrid. Recuperado el 25 de mayo de 2011. Desde www.textoenlinea.com.ar.

Frias, Xavier (2002) Introducción a la psicolingüística. Iahua. Revista Pshilologica Romanica. Suplemento 06. Recuperado el 20 de Julio de 2009 desde: www.romaniaminornet/ianua.

García Santa-Cecilia, A. (2004) La enseñanza del español como lengua extranjera desde la perspectiva del Marco Común Europeo de Refer $\neg$ encia. Congresos Internacionales de la Lengua Española. Congreso de Rosario, Argentina. Extraído el 26 de diciembre. Desde: http://www. congresosdelalengua.es/accesibilidad.htm.

García Vidal, Jesús y colab. (2000) Batería Psicopedagógica Evalúa 5. Instituto de Orientación Psicológica EOD. Madrid.

Genesse, F. (2004) What do we know about bilingual education for majority language students. Handbook of bilinguaslim and multilingualism. Malden MA. Black well.

Genesse, F. (2007) ¿Qué es ser bilingüe? Revista Internacional Magisterio. Educación y Pedagogía. Bogotá.

Gilboy, Elizabeth (1983) La adquisición del lenguaje en niños bilingües. Revista Infancia y Aprendizaje Nro. 21. España. Extraído el 30 de junio de 2009. Desde: www.dialnet.unirioja.es.

Godenzzi, Juan Carlos. (2001) I Coloquio tres espacios lingüísticas ante los desafíos de la mundialización. Paris Marzo. Recuperado de: www.campus.oei.org. El: 13 de setiembre de 2011. 
Gómez, Maigualida y Pérez, Edith. (2006) El desarrollo cognitivo en niños bililingües. Universidad Simón Bolívar. Venezuela.

González José Luis y Asensio, Mabel (1998) Las estrategias de aprendizaje en la instrucción de lenguas extranjeras. Revista Estudios de Psicología Nro. 60. Recuperado el 01 de septiembre de 2009. Desde: www.dialnet.unirioja.es.

González Moreyra, Raúl (1995) Exploración del desarrollo del lenguaje en el niño peruano menor de tres años: Un modelo interactivo. Proyecto de Innovaciones Pedagógicas No Formales. Bernad Van Leer. Ministerio de Educación, Lima.

González Moreyra, Raúl y colab. (2001) Lectura comprensiva temprana. Para que leamos desde la infancia, mucho más y mejor. Serie: Formación docente y modernización de la Educación 5. Ministerio de Educación y GTZ Corporación Técnica Alemana. Lima

Grant. (1996). Dirección estratégica. Conceptos, técnicas y aplicaciones. Madrid: Civitas.

Grosjean, F. (1985) The Bilingual as a competent buy specific speaker-hearer. Journal of multilingual and multicultural development.

Guerrero, Gabriela. (2005) Un análisis de la implementación de la política nacional de educación bilingüe intercultural en Puno. Tesis de Maestría. Estudios de desarrollo con especialización en políticas públicas en el Institute of Social Studies en La Haya. Países Bajos.

Hagene, Claude (1996) Lṛnfant aux deux langues. Editions. Jacob. Paris.

Hammers, J y Blanc, M. (2000) Bilinguality and Bilingualism. Cambridge: Cambridge University Press.

Harley, Birgit. (1990). The development of second language proficiency. Cambridge. University Press.

Huguet Canalís, Angel. (1994) Conocimiento lingüístico de los escolares de la franja oriental de Aragón e implicaciones curriculares del tratamiento de las lenguas. Universidad de Lleida.

Huguet Canalís, Angel (2003) Lenguaje y Rendimiento Escolar, un estudio sobre las relaciones entre el conocimiento lingüístico y matemático en el contexto bilingüe asturiano. Academia Llingua Asturiana.

Hymes, D. (1971) Competence and performance in linguistic theory Acquisition of languages: Models and methods. Ed. Huxley and E. Ingran. New York. Academic Press.

Hymes, D. (1995) Acerca de la competencia comunicativa. Documentos básicos en la enseñanza de lenguas extranjeras. M Llobera. Edelsa Didascalia. Madrid.

Krashen, S. y Terrell, T. (1983) The Natural Approach: Language Acquisition in the Classroom. Oxford: Pergamon.

Lebrnn Fuentes, Antonio (2009) Teorías generales sobre el aprendizaje y la adquisición de una lengua extranjera. Temas para la educación. Recuperado el 20 de septiembre de 2009. Desde: www.fe.ccooes/andalucia/docu. 
López García, Angel (1999) Lingüística General y Aplicada. Colección Educación y Materiales. Universidad de Valencia.

Lozano Vallejo, Ruth (1999) Reseña de políticas para poblaciones indígenas en el Perú. Educación bilingüe intercultural. Experiencias y propuestas. Primer Congreso Nacional de Educación Bilingüe Intercultural. Huancayo.

Lupiañez, Jy Rico, L. (2006) Análisis didáctico y formación inicial de profesores: organización de competencias y capacidades de los escolares en el caso de los números de decimales. Universidad de Granada.

Luque, Beatriz (2008) Enfoques Actuales usados en la enseñanza de segundas lenguas. Letras. V. 50. Nro. 76. Recuperado el 07 de julio de 2009. Desde: www.scielo.cl.

Lyster, R. (2007) Learning and Teaching Languages throughContent.Amsterdam: John Benjamins.

Manriquez Leonardo y Acle, Guadalupe (2006) Bilingüismo y competencia lingüística. Diferencias en las subcompetencias lingüísticas Nahuatl-Español. Revista Interamericana de Psicología Vol. 40. N. ${ }^{\circ}$ 3. México. Recuperado el 29 de junio de 2009. Desde: www. psicorip.org.

Martínez Agudo, Juan de Dios (1999) Reflexiones psicolingüísticas sobre la naturaleza y dinámica del fenómeno de la interferencia durante los procesos de aprendizaje y adquisición de una lengua extranjera. Universidad de Extremadura. Recuperado el 23 de junio de 2009. Desde: www. dialnet.unirioja.es.

Martí y colab. (2007) Palabras y mundos. Informe sobre las lenguas del mundo. Icaria Editorial SA. Barcelona.

Martínez, Leonardo y Acle, Guadalupe (2006) Bilingüismo y competencia lingüística diferencias de las subcompetencias lingüísticas Nahuatl-Español. Universidad Autónoma de México. Vol 40. N. ${ }^{\circ} 3$.

Mayor, Juan. (1993) Problemas y métodos de enseñanza del español como Lengua extranjera. IV. Congreso Internacional de ASELE. Extraído el 10 de enero 2011. Desde www.cvc.cervantes. es. Madrid.

Mayor, Juan. (1994) Adquisición de una segunda lengua. Asele. Actas IV. Extraído el 10 de enero 2011. Desde www.cvc.cervantes.es. Madrid.

Mediano, Nancy. (1973) Educación Indígena en los Altos de Chiapas. Instituto Nacional Indigenista. Recuperado el 07 de julio de 2009. Desde: www.latarea.com.mx.

Mercau María Virginia. (2007) La enseñanza de inglés en las escuelas primarias: Propósito o despropósito. Memorias del XXI Foro de Especialistas Universitarios en Lenguas Extranjeras.

Ministerio de Educación Nacional República de Colombia (2006) Formar en lenguas extranjeras: el reto: serie guías N. ${ }^{\circ} 22$. Estándares básicos de competencias en lenguas extranjeras: inglés.

Ministerio de Educación del Perú (2011) El enfoque intercultural, bilingüe y la participación comunitaria en el proceso de diversificación curricular. Dirección General de Educación Intercultural Bilingüe y Rural. Lima. 
Morales, G. (1996) El giro cualitativo de la educación. Cali. XYZ. Editores.

Moreno Fernandez, Francisco (2009) Principios de sociolinguistica y sociología del lenguaje. Ed. Ariel. España.

Muñoz Redondo, M. Carmen y López Bautista, Dolores (2002-2003) Aprendizaje Temprano de una segunda lengua. Encuentro Revista de investigación e innovación en la clase de idiomas. Madrid.

Muñoz, Carmen y colab. (2003) En torno a los efectos de la edad en el aprendizaje escolar de una lengua extranjera. Universidad de Barcelona. Recuperado el 27 de abril de 2011. Desde: http:// www.aulaintercultural.org.

Numan, D. (1989) Designing talks for the communicative classrooms. Cambridge: Cambridge University Press.

O'Shanahan, Isabel y colab. (2010) Analizando procesos cognitivos y de escritura en niños hispano-parlantes que aprenden inglés como segunda lengua y niños canadienses de habla inglesa. European Journal of Education and Psychology. Editorial. CENFINT. España.

Pilleux, Mauricio. (2001) Competencia comunicativa y análisis del discurso. Estud.filol. Nro 36. Recuperado el 08 de julio de 2009. Desde: www.scielo.cl.

Porto Riobo, Ana María (1991) Análisis diferencial de las habilidades psicolingüísticas en alumnos de rendimiento alto y bajo. Ministerio de Educación y Ciencia. INCE. España. Recuperado el 21 de julio de 2009 de: www.doredin.mec.es.

Rodriguez Romero, Bony (2001) Problemas en la adquisición y aprendizaje de la lecto-escritura en una segunda lengua y resultados del plan de tutoría especialmente aplicado. Tesis para obtener el grado de Magister en Educación. Universidad Femenina del Sagrado Corazón. Lima.

Roldan Tapia, Antonio (2000) El aprendizaje centrado en el alumno: de la teoría a la práctica. I.E.S. Alhaken II/Universidad de Córdoba Extraído el 08 de abril 2011 Desde: www.encuentrojournal. org/textos.

Romaine, Suzzane. (1995) Bilingualism. Blackwell Publishers Inc. Massachussetts.

Román Pérez, Martiniano (2005) Aprender a aprender en la sociedad del conocimiento. Universidad de Concepción. Facultad de Educación. Dirección de Educación Continua. Chile.

Romero Aguilar, Laura (2008) Tendencias actuales en la investigación diacrónica de la lengua. Actas VIII del Congreso Nacional de la Asociación de jóvenes e investigadores de historiografía e historia de la lengua española. Barcelona.

Rosas, R. y Sebastián, C. (2001) Piaget, Vigotski y Maturana. Constructivismo a tres voces. Buenos Aires: Aique Group.

Salaberry Ramiro, María Sagrario (2007) Modelos de Bilingüismo. Departamento de Filología Inglesa y Alemana. Universidad de Almería. Recuperado el 26 de julio de 2009. Desde: www. redes.cepalcala.org.

Sánchez López, María del Pilar y Rodríguez de Tembleque, Rosario (1998) El bilingüismo, bases 
para la intervención psicológica. Personalidad, Evaluación y Tratamiento Psicológico. Síntesis Psicología. Madrid.

Sarto, Pilar (1997) El bilingüismo una aportación a las necesidades educativas lingüísticas. Amaru Ediciones. Salamanca.

Savignon, S. J. (1991) Communicative language teaching: State of the art. TESOL Quarterly.

Shaffer, David (2000) Psicología del desarrollo: Infancia y Adolescencia. International Thomson Editores. México.

Signoret, Docasberro (2003) Bilingüismo y cognición ¿Cuándo iniciar el bilingüismo en el aula? Perfiles educativos Vol XXV. Nro. 102. Mṛxico. Recuperado el 25 de septiembre de 2009. Desde http:/redalyc.uaemex.mx.

Siguán, M. y Mackey, W. F. (1986) Educación y bilingüismo. Madrid: Santillana/Unesco.

Silva D y Simonsen, E. (2007) La difícil tarea de enseñar matemática en un segundo idioma. Colegio Inglés British Royal School. Concepciṇn. La Tercera. Santiago de Chile.

Sinclair. (1985) La adquisición del lenguaje desde el punto de vista piagetino. En Varios Psicolingüística Genética. Barcelona.

Tardo Fernández, Yaritza (2005) Estrategias de comunicación y desarrollo de la autonomía en las clases de Español como Lengua Extranjera (ELE): ¿moda o necesidad? Departamento de Idiomas Facultad de Humanidades. Universidad de Oriente Santiago de Cuba.

Titone, Renzo (1981) Psicodidáctica. Narcea. España.

Titone, Renzo (1976) Bilingüismo y educación. Barcelona, Fontanela

Tudor, I. (1996) “30 years of leamer-centredness”, GRETA, Revista para Profesores de Inglés 4/2.

Urrutia, Hernán, Luis Candia, María D. Martínez y Francisco Milla (1998) Bilingüismo y rendimiento académico en la Comunidad Autónoma Vasca. Estudio de resultados de la educación bilingüe. Bilbao: Jóvenes por la Paz. 1998. Universidad de Tarapacá Departamento de Español. Arica

Velázquez Martínez, María Patricia (2005) Breve Introducción al Fenómeno del Bilingüismo en México. Universidad de Guadalajara. México. Recuperado el 01 de julio de 2009. Desde: www. sincronía.cucsh.udg.mx.

Vigotsky, L. (1995) Pensamiento y lenguaje. Buenos Aires: Ediciones Fausto.

Villaalba, F y Hernández MT. (1999). Concepto de lengua y teorías de aprendizaje en el enfoque comunicativa de idiomas. En Cuaderno de Bitácora. Revista del CPR De Parla. N. ${ }^{\circ}$ 3. Madrid.

Villaalba, F y Hernández MT. (2008) La enseñanza de una segunda lengua y la integración del currículo en el I. Ballano I Jornada sobre Lenguas, Currículo y Alumnado Inmigrante. Bilbao U. Deusto.

Volterra, Ty Taeschner, V. (1983) La adquisición y el desarrollo del lenguaje en niños bilingües. Revista Infancia y Aprendizaje. Recuperado el 10 de setiembre de 2010. Desde: www.dialnet.unirioja.es. 
Woolfolk, Anita (2006) Psicología Educativa. Prentice Hall. 1999. México.

Zavala, Virginia (2001) Oralidad y escritura en la educación bilingüe (a propósito de interculturalidad). Lima: Ministerio de Educación, Programa Especial MECEP, Cooperación Técnica Alemana, GTZ, Cooperación Financiera, República Federal Alemana, KFW (Serie: Educación Bilingüe Intercultural).

Fecha de recepción: 07-06-17

Fecha de aceptación: 14-09-17 